\title{
The Relationship between Food Intake and Respiratory Quotient in Mice
}

\author{
By A. D. DEWAR AND W. H. NEWTON \\ Physiological Laboratory, Department of Physiology and Histology, \\ University of Liverpool
}

(Received 31 March 1948)

It is a generally accepted principle that the respiratory quotient is influenced by the composition of the food eaten, but the effect on it of the quantity of food seems to have been neglected. Occasional papers can be found which contain data showing an increase in R.Q. at higher intakes (e.g. Williams, Riche \& Lusk, 19r2; Forbes, Swift, Elliott \& James, 1946), but the levels used are too few to justify quantitative inferences.

One reason for this omission is undoubtedly that the field has been dominated by the important conception of 'basal metabolism', so that most studies have been carried out on animals in the post-absorptive state, and periods of observation have tended to be short. The evidence for a quantitative relationship between R.Q. and food intake which is presented in this paper is derived from observations made over $24 \mathrm{hr}$. periods on animals provided with food and drink.

\section{METHODS}

The procedure is described in the preceding paper (Dewar \& Newton, 1948), and was applied to two albino mice. The experiments on mouse no. 4 covered 6 weeks and included eighteen periods of $24 \mathrm{hr}$., some of which were isolated while others were consecutive; the longest run was 8 days. Mouse no. 5 was studied over a period of 3 weeks, ten 24 hr. experiments being conducted separately or in runs of up to 4 days.

Diet MET 2 (Dewar \& Newton, 1948) was used throughout and, except for a single day's experiment (mouse no. 4) when food was entirely withheld, was available ad lib. The remaining variations in the quantities eaten were voluntary on the part of the mice.

The approximate non-protein R.Q. of the diet was determined in a simple feeding experiment on six mice, lasting 4 days, in which it was found that of $x \cdot 707 \mathrm{~g}$. carbohydrate and $0.308 \mathrm{~g}$. fat eaten, 0.055 and $0.003 \mathrm{~g}$. respectively were lost as faeces. The net available carbohydrate was therefore $1.652 \mathrm{~g}$. and the net available fat $0.305 \mathrm{~g}$. Combustion of these quantities would produce an R.Q. of 0.909. Analysis of the more scanty material from the two mice under experiment yielded values of 0.909 (mouse no. 4) and 0.910 (mouse no. 5).

\section{RESULTS}

In Fig. I the quantity of food eaten during each experimental period is plotted against the non-protein R.Q. for the period. On the assumption that the relationship is linear, the equation of the best straight line relating the points has been calculated. The 
Vol. 2

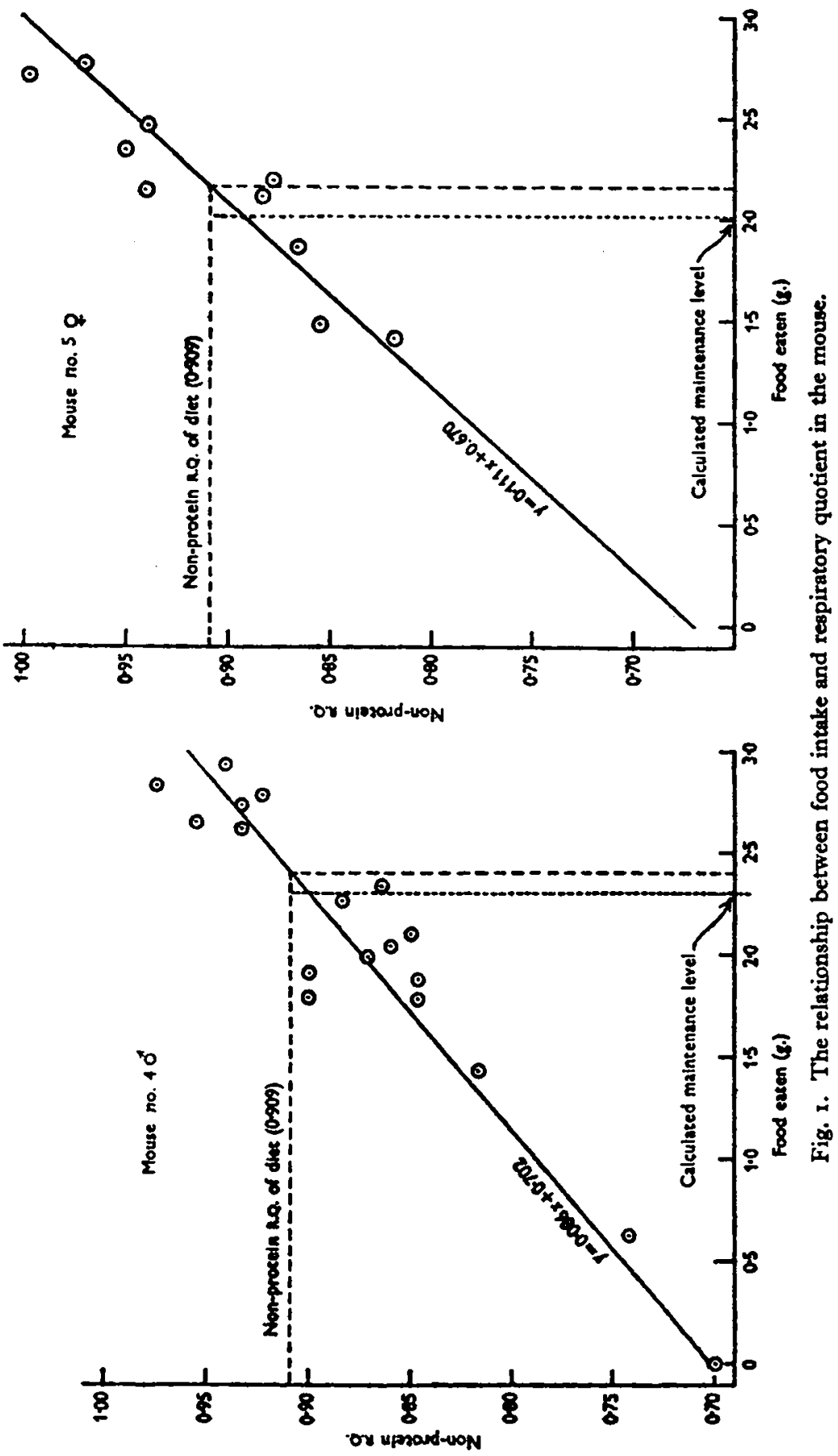


scatter of the points may be partly due to the assumption that the food eaten and the food assimilated are the same, whereas the amount of food in the animal's alimentary tract is not necessarily the same at the beginning and end of an experiment. The results for mouse no. 5 are of less significance than those for mouse no. 4 owing to the fewer observations and to the more limited range of levels of intake.

Two interesting facts are immediately evident from the intake-R.Q. curves. First, the intercept of the line on the ordinate is approximately the R.Q. of fat. Secondly, if the intake levels which would correspond to the calculated non-protein R.Q. of the diet are read off on the graphs, they are found to be very close to the weights of food required daily by the respective mice for maintenance. Thus, the maintenance levels for mice nos. 4 and 5 are, respectively, 2.296 and 2.013 g. of diet MET 2, and the corresponding figures obtained from the graphs are 2.407 and $2.153 \mathrm{~g}$.

The maintenance requirement of each mouse is calculated by dividing the average energy expenditure by the calorific value of the diet for the mouse (i.e. the physiological energy value of the food less that of the faeces), during experiments in which no marked loss of weight occurs (see Dewar \& Newton, 1948). Thus:

Average energy expenditure (Cal./24 hr.)

Calorific value of food (Cal./g.)

$\therefore$ maintenance requirement (g./24 hr.)

$\begin{aligned} & \text { Mouse no. } 4 \\ & 9 \cdot 69 \mathrm{r} \\ & 4.220 \\ = & \frac{9 \cdot 69 \mathrm{I} \mathrm{Cal} / 24 \mathrm{hr} .}{4.220 \mathrm{Cal} . / \mathrm{g} .} \\ = & 2.296\end{aligned}$

Mouse no. 5

8.630

4.286

$\frac{8.630 \mathrm{Cal} . / 24 \mathrm{hr}}{4 \cdot 286 \mathrm{Cal} . / \mathrm{g} .}$

$2 \cdot 013$

The calorific value of the food differs slightly in the two mice owing to relative differences in the quantities of faeces passed. The calorific values of both food and faeces were determined indirectly by multiplying the weights of the constituent foodstuffs by the following equivalents in Cal./g.: carbohydrate (starch) $4 \cdot 2$, fat 9.3 , protein (casein) 4.59 (Kriss \& Miller, 1934).

\section{DISCUSSION}

The interpretation of the results agrees with generally accepted principles concerning the utilization of foodstuffs, and the chief feature of the experiments is that these principles appear to be quantitatively demonstrated. At the level of food intake required for maintenance, the animal's R.Q. is the same as that of the diet, i.e. the foodstuffs are being burnt in the same proportion as that in which they are presented. Below this level the R.Q. decreases as the animal progressively draws upon its fat reserves, until at zero intake the R.Q. becomes that of fat. Above the maintenance level, the quotient increases in proportion to the food intake. Either the carbohydrate of the diet is being progressively preferred as the source of fuel and the fat stored, or, alternatively, fat and carbohydrate are being burned in the proportions in which they are present in the food, but the excess carbohydrate ingested is converted into fat and stored along with the corresponding excess of ingested fat. Either possibility must lead to the same net result and the same R.Q. 
From the wide range of R.Q. exhibited in these experiments, it is clear that the composition of the food alone permits no anticipation of the animal's R.Q. However, if, in addition to the composition of the food, the quantity eaten is known, and the relationship which these results demonstrate is used, it would seem that a reasonably accurate prediction of the R.Q. can be made.

\section{SUMMARY}

I. Determinations over $24 \mathrm{hr}$. periods of the respiratory exchange of mice which have access to food revealed a linear relationship between non-protein R.Q. and quantity of food eaten.

2. At the maintenance level of food intake the observed non-protein R.Q. was that of the diet; below this level the R.Q. progressively declined as body fat was increasingly utilized until, at zero intake, it approximated to that of fat; above the maintenance level the R.Q. increased, owing probably to conversion of carbohydrate to fat or to progressive preferment of carbohydrate as fuel.

Our thanks are due to the Medical Research Council for assistance in defraying the expenses of this work.

\section{REFERENCES}

Dewar, A. D. \& Newton, W. H. (1948). Brit. Y. Nutrit. 2, 123.

Forbes, E. B., Swift, R. W., Elliott, R. F. \& James, W. H. (1946). J. Nutrit. 31, 213.

Kriss, M. \& Miller, R. C. (1934). Ұ. Nutrit. 8, 669 .

Williams, H. B., Riche, J. A. \& Lusk, G. (1912). Э. biol. Chem. I2, 349. 